The Astrophysical Journal, 566:387-391, 2002 February 10

(C) 2002. The American Astronomical Society. All rights reserved. Printed in U.S.A.

\title{
SIGMA OBSERVATIONS OF THE BURSTING PULSAR GRO J1744-28
}

\author{
J. Mejía AND T. VILlela \\ Instituto Nacional de Pesquisas Espaciais, Divisão de Astrofísica, C.P. 515, 12201-970, São José dos Campos, SP, Brazil; mejia@das.inpe.br \\ P. GOLDONI AND F. LEBRUN \\ CEA/DSM/DAPNIA/SAp, CEA-Saclay, F-91191 Gif-sur-Yvette Cedex, France \\ L. BOUCHET, E. JourdaIn, J.-P. RoQUES, AND P. MANDROU \\ Centre d'Etude Spatiale des Rayonnements, 9 Avenue du Colonel Roche, B.P. 4346, 31029 Toulouse Cedex, France
}

AND

\begin{abstract}
S. Kuznetsov, N. Khavenson, A. Dyachkov, I. Chulkov, B. Novikov, K. Shuhanov, I. Tserenin, and A. Sheikhet Space Research Institute, Profsoyuznaya 84/32, Moscow 117810, Russia Received 2001 September 4 ; accepted 2001 October 11
\end{abstract}

\begin{abstract}
We present the results of the GRANAT/SIGMA hard X-/soft $\gamma$-ray long-term monitoring of the Galactic center (GC) region concerning the source GRO J1744-28, discovered on 1995 December 2 by CGRO/BATSE. SIGMA observed the region containing the source in 14 opportunities between 1990 and 1997. In two of these observing sessions, corresponding to 1996 March and 1997 March, GRO J1744-28 was detected with a confidence level greater than $5 \sigma$ in the $35-75 \mathrm{keV}$ energy band without detection in the $75-150 \mathrm{keV}$ energy band. For the other sessions, upper limits of the flux are indicated. The particular imaging capabilities of the SIGMA telescope allow us to identify, specifically, the source position in the very crowded GC region, giving us a mean flux of $(73.1 \pm 5.5) \times 10^{-11}$ and $(44.7 \pm 6.4) \times 10^{-11}$ ergs $\mathrm{cm}^{-2} \mathrm{~s}^{-1}$ in the 35-75 keV energy band, for the 1996 March and 1997 March observing sessions, respectively. Combining the 1997 March SIGMA and BATSE observations, we found evidence pointing to the type II nature of the source bursts for this period. For the same observing campaigns, spectra were obtained in the 35 to $150 \mathrm{keV}$ energy band. The best fit corresponds to an optically thin thermal Bremsstrahlung with $F_{50 \mathrm{keV}}=3.6( \pm 0.6) \times 10^{-4}$ photons $\mathrm{cm}^{-2} \mathrm{~s}^{-1} \mathrm{keV}^{-1}$ and $k T_{\text {Bremss }}=28 \pm 7 \mathrm{keV}$ for the first campaign, and $F_{50 \mathrm{keV}}=2.3( \pm 0.7) \times 10^{-4}$ photons cm $\mathrm{cm}^{-2} \mathrm{~s}^{-1} \mathrm{keV}^{-1}$ and $k T_{\mathrm{Bremss}}=18_{-7}^{+12} \mathrm{keV}$ for the second. This kind of soft spectrum is typical of binary sources containing a neutron star as the compact object, in contrast to the harder spectra typical of systems containing a black hole candidate.
\end{abstract}

Subject headings: Galaxy: center - pulsars: individual (GRO J1744-28) - X-rays: bursts

\section{INTRODUCTION}

GRO J1744-28, the bursting pulsar, was discovered on 1995 December 2 by CGRO/ BATSE (Fishman et al. 1995; Kouveliotou et al. 1996). Timing studies revealed a period of $0.467 \mathrm{~s}$ in the persistent flux and an 11.8 day orbital period (Finger, Wilson, \& van Paradijs 1996). This source presented two strong outbursts: at the end of 1995 and early 1996, and then almost a year later. At that time, this was the only source known to show, simultaneously, periodic X-ray pulsations and frequent X-ray bursts. The presence of pulsations indicates that the compact object is a neutron star. The X-ray mass function, determined by Finger et al. (1996) as being $f\left(M_{\mathrm{X}}\right)=1.31 \times 10^{-4} M_{\odot}$, together with a mass canonical value for the compact object of $1.4 M_{\odot}$, suggest that the neutron star in GRO J1744-28 is being fed by Roche lobe overflow from a low-mass red giant (Daumerie et al. 1996; Miller 1996; Sturner \& Dermer 1996; Bildsten \& Brown 1997).

The best position coordinates for the source are those obtained from triangulation of the Ulysses and BATSE observations of the region. With this method, Hurley et al. (2000) determined the coordinates R.A. $=17^{\mathrm{h}} 44^{\mathrm{m}} 32^{\mathrm{s}}$, decl. $=-28^{\circ} 44^{\prime} 31^{\prime \prime}$.7 (J2000.0). A possible infrared counterpart was reported by Augusteijn et al. (1996), while a posterior optical/IR observation of the same region by Cole et al.
(1997) neither confirmed nor discarded this IR counterpart. Based on the $A S C A$ data, the absorption column, $N_{\mathrm{H}}$, for the position of GRO J1744-28 was determined as being (5-6) $\times 10^{22} \mathrm{~cm}^{-2}$, independent of the emission date and phase and, therefore, corresponding to interstellar absorption (Dotani et al. 1996; Nishiuchi et al. 1999). This suggests that the source is located near the Galactic center (GC), at a distance of $\sim 8.0 \mathrm{kpc}$. The source was also observed with GRANAT/WATCH from 1996 January 13 to March 12 (Sazonov, Sunyaev, \& Lund 1997) showing, at the maximum between bursts, a daily average flux of $\sim 3.7 \mathrm{crab}$, in the 8-20 keV energy band.

Between 1990 and 1997, the SIGMA telescope was pointed in the direction of the GC region two times per year, in the March-April and September-October periods. In 14 opportunities, the region containing GRO J1744-28 was observed, for a total of $\sim 2993 \mathrm{hr}$. In two of these sessions, corresponding to 1996 March and 1997 March, the source was clearly visible, with a confidence level higher than $5 \sigma$. In this paper, the analysis of these observations is presented. Also, upper limits are reported for the remaining observing sessions. Finally, for the 1997 March observing session, a combination of the SIGMA value for the integrated flux of the source with the BATSE value for the burst mean flux allows us to obtain evidence favoring the type II 

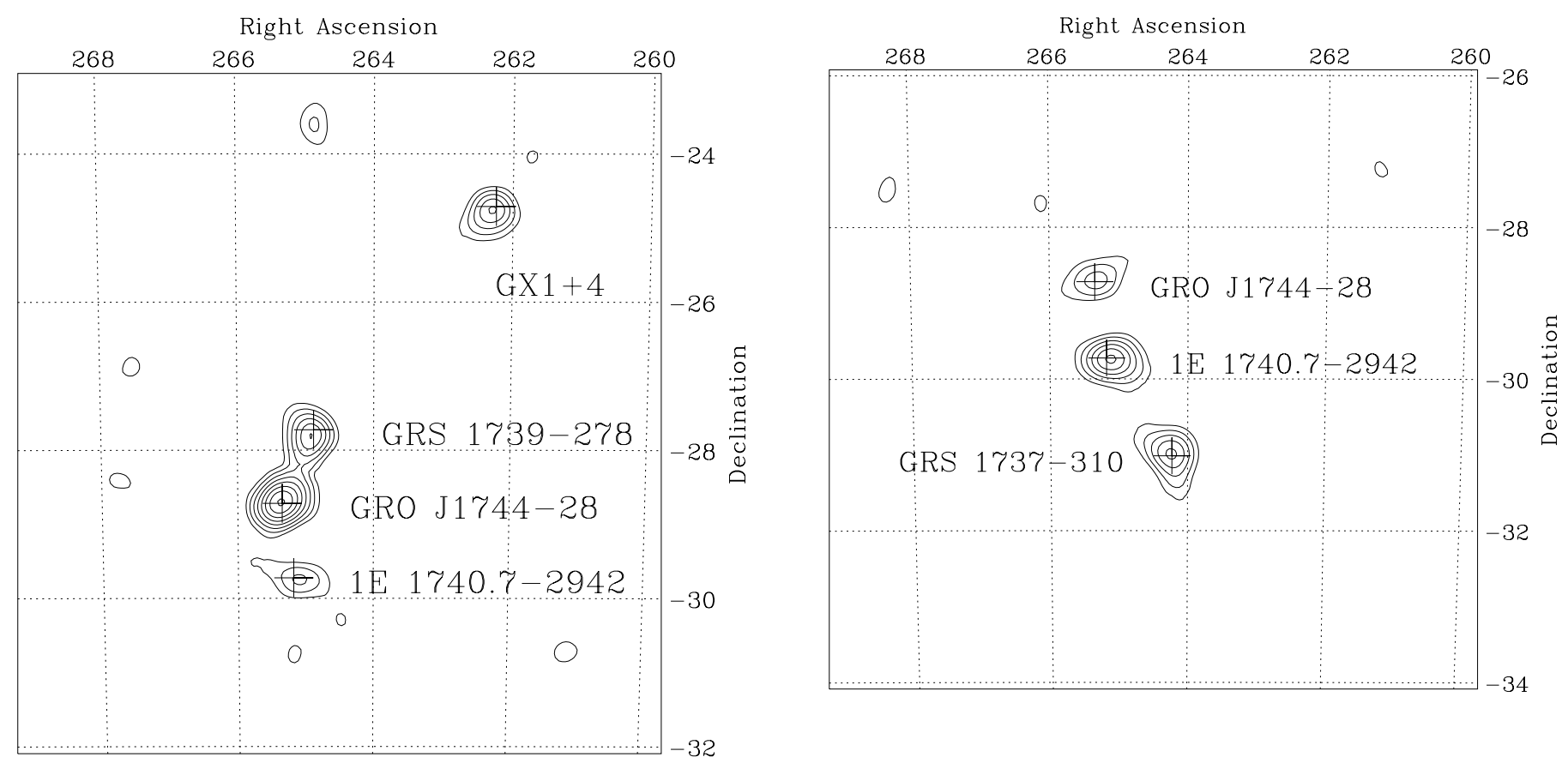

FIG. 1.-Contour images of the GC region obtained with SIGMA on 1996 March (left) and 1997 March (right) in the 35-75 keV energy band. Right ascension and declination are in degrees. Notice the presence of the transient source GRS 1737-31 in the 1997 March image. Coordinates are for 1950.0 . Confidence levels start at $3 \sigma$ with $1.5 \sigma$ steps.

nature of the bursts, due to spasmodic accretion rather than thermonuclear burning of matter.

\section{THE SIGMA TELESCOPE}

The French coded-mask telescope SIGMA (Paul et al. 1991) on board the Russian GRANAT orbital observatory provides high-resolution images in the hard X-ray/soft $\gamma$-ray band from 35 to $1300 \mathrm{keV}$, divided in 95 energy channels. The nominal angular resolution of the instrument is $\sim 15^{\prime}$. The position determination accuracy of the instrument is $3^{\prime}-5^{\prime}$ for a $6 \sigma$ source and less than $1^{\prime}$ for a $30 \sigma$ one. The fully coded field of view of the instrument is a $4.7 \times 4.3$ rectangle, while the half-sensitivity boundary of the field of view corresponds to an $11.5 \times 10^{\circ} .9$ rectangle. The energy

TABLE 1

SIGMA 1996 March AND 1997 March ObSERVATION Log OF the Galactic

CENTER

Region Containing the COORDINATES OF GRO J1744-28

\begin{tabular}{|c|c|c|c|c|}
\hline Session & $\begin{array}{l}\text { Date } \\
\text { (UT) }\end{array}$ & $\begin{array}{l}\text { Exposure } \\
\text { (hr) }\end{array}$ & $\begin{array}{l}\text { 35-75 keV Flux } \\
\text { (mcrab) }\end{array}$ & $\begin{array}{c}\text { 75-150 keV Flux } \\
\text { (mcrab) }\end{array}$ \\
\hline \multicolumn{5}{|c|}{1996 March Campaign } \\
\hline $894 \ldots \ldots \ldots$ & $15.63-16.68$ & 25.25 & $80 \pm 26$ & $<34$ \\
\hline $895 \ldots \ldots \ldots$ & $16.82-18.23$ & 33.84 & $77 \pm 15$ & $<23$ \\
\hline $896 \ldots \ldots \ldots$ & $18.92-20.38$ & 35.06 & $62 \pm 15$ & $<25$ \\
\hline $897 \ldots \ldots \ldots$ & $20.77-22.48$ & 41.14 & $75 \pm 16$ & $<21$ \\
\hline $898 \ldots \ldots \ldots$ & $23.52-24.52$ & 24.03 & $106 \pm 18$ & $57 \pm 28$ \\
\hline $899 \ldots \ldots \ldots$ & $24.77-26.48$ & 41.14 & $86 \pm 16$ & $<24$ \\
\hline $900 \ldots \ldots \ldots$ & $27.60-28.66$ & 25.43 & $96 \pm 20$ & $<30$ \\
\hline $901 \ldots \ldots \ldots$ & $28.79-30.50$ & 41.14 & $96 \pm 17$ & $<28$ \\
\hline Total...... & & 267.03 & $85 \pm 6.4$ & $<28$ \\
\hline \multicolumn{5}{|c|}{1997 March Campaign } \\
\hline $935 \ldots \ldots \ldots$ & $14.54-15.59$ & 25.30 & $34 \pm 17$ & $<30$ \\
\hline $937 \ldots \ldots \ldots$ & $18.68-19.65$ & 23.45 & $75 \pm 22$ & $<29$ \\
\hline $938 \ldots \ldots \ldots$ & $19.79-20.69$ & 21.49 & $69 \pm 21$ & $<33$ \\
\hline $939 \ldots \ldots \ldots$ & $22.47-23.58$ & 26.66 & $43 \pm 17$ & $<28$ \\
\hline $940 \ldots \ldots \ldots$ & $23.71-24.71$ & 24.00 & $22 \pm 21$ & $<28$ \\
\hline $941 \ldots \ldots \ldots$ & $26.49-27.61$ & 26.81 & $83 \pm 18$ & $46 \pm 26$ \\
\hline $942 \ldots \ldots \ldots$ & $27.73-28.63$ & 21.58 & $34 \pm 20$ & $58 \pm 31$ \\
\hline Total...... & & 148.53 & $52 \pm 7.4$ & $<33$ \\
\hline
\end{tabular}

NoTE. -1 mcrab corresponds to $\sim 1.08 \times 10^{-4}$ and $\sim 4.4 \times 10^{-5}$ photons $\mathrm{cm}^{-2}$ $\mathrm{s}^{-1}\left(\sim 8.6 \times 10^{-12}\right.$ and $\left.\sim 7.3 \times 10^{-12} \mathrm{ergs} \mathrm{cm}^{-2} \mathrm{~s}^{-1}\right)$ for the $35-75 \mathrm{keV}$ and $75-150$ $\mathrm{keV}$ bands, respectively. 
resolution of the instrument at $511 \mathrm{keV}$ is $\sim 8 \%$. The typical duration of individual observing sessions is $\sim 20 \mathrm{hr}$, which provides a $1 \sigma$ sensitivity of $\sim 20$ mcrab in the $35-150 \mathrm{keV}$ energy domain. For details on the SIGMA in-orbit performances, see Bouchet et al. (2001).

\section{THE OBSERVATIONS}

The region containing the source GRO J1744-28 was observed by SIGMA in 14 different opportunities, from 1990 to 1997. In two of these observing campaigns, the source was clearly detected in the 35-75 keV energy band, with a confidence level higher than $5 \sigma$, as shown in Figure 1. In contrast, the source was not detected in the 75-150 $\mathrm{keV}$ energy band. The first campaign began on $1996 \mathrm{March}$ 15 (MJD 50157.63), 103 days after the discovery of the source by BATSE, and lasted until March 30, corresponding to $\sim 267 \mathrm{hr}$ of effective observing time (see Table 1). In this period, GRO J1744 -28 appears as a $13.5 \sigma$ source with a mean detected flux in the $35-75 \mathrm{keV}$ energy band of $85 \pm 6.4$ mcrab $\left[7.3( \pm 0.6) \times 10^{-10}\right.$ ergs $\left.\mathrm{cm}^{-2} \mathrm{~s}^{-1}\right]$, showing a soft spectrum that can be approximated by an optically thin thermal Bremsstrahlung model $(\alpha=-1.4)$ with a flux at $50 \mathrm{keV}$ of $F_{50 \mathrm{keV}}=3.6( \pm 0.6) \times 10^{-4}$ photons $\begin{array}{cccc}\mathrm{cm}^{-2} & \mathrm{~s}^{-1} & \mathrm{keV}^{-1} & \text { and } \\ & k T_{\text {Bremss }}=28 \pm 7 & \mathrm{keV}\end{array}$ $\left[\chi^{2}\right.$ (d.o.f.) $\left.=13.0(8)\right]$.

The second campaign began on 1997 March 14 (MJD 50521.31) and last until March 28, corresponding to an effective observational time of $\sim 148.5 \mathrm{hr}$. In this opportunity, the mean flux of the source in the $35-75 \mathrm{keV}$ energy band was of $52 \pm 7.4 \mathrm{mcrab}\left[4.5( \pm 0.6) \times 10^{-10}\right.$ ergs $\mathrm{cm}^{-2} \mathrm{~s}^{-1}$ ], corresponding to a confidence level of $8 \sigma$. The source showed a very steep spectrum fitting with an optically thin thermal Bremsstrahlung model with a $F_{50 \mathrm{keV}}=2.3( \pm 0.7) \times 10^{-4}$ photons $\mathrm{cm}^{-2} \mathrm{~s}^{-1} \mathrm{keV}^{-1}$ and $k T_{\text {Bremss }}=18_{-7}^{+12} \mathrm{keV}\left[\chi^{2}\right.$ (d.o.f.) $\left.=8.04(8)\right]$. In Table 1, the values of the fluxes corresponding to each one of the sessions of these two campaigns in the $35-75 \mathrm{keV}$ and $75-150$ $\mathrm{keV}$ energy bands are indicated.

\section{THE LIGHT CURVE AND SPECTRA OF GRO J1744-28}

In Figure 2, the light curves of the source GRO J1744-28 for the campaigns corresponding to 1996 March and 1997 March are presented. In the first campaign, the source flux in the $35-75 \mathrm{keV}$ energy band was almost con- stant with an average flux of $\sim 85$ mcrab or $7.3 \times 10^{-10}$ ergs $\mathrm{cm}^{-2} \mathrm{~s}^{-1}$. The second campaign does not show significant flux variations; the average flux being $\sim 52$ mcrab or $4.5 \times 10^{-10}$ ergs $\mathrm{cm}^{-2} \mathrm{~s}^{-1}$ (see also Trudolyubov et al. 1999). This last value is a factor 7 lower than that reported by Woods et al. (1999) of $\sim 3 \times 10^{-9} \mathrm{ergs} \mathrm{cm}^{-2} \mathrm{~s}^{-1}$, as taken from the BATSE observations of the source using the Earth-occultation technique in the 30 to $100 \mathrm{keV}$ energy band.

Since GRO J1744-28 is located near the GC, it was within the SIGMA field of view during all the observations constituting the GC survey conducted by SIGMA between 1990 and 1997, which allows us to estimate upper limits for the hard X-ray flux from the source position before and after the 1996 and 1997 outbursts. An analysis of these SIGMA observations was done in order to verify the possibility of source appearances in other epochs than that corresponding to the two outbursts on 1996 and 1997. In Figure 3, the light curve of the source is shown, corresponding to all the pointed SIGMA observations of the GC region containing the source, in the $35-75 \mathrm{keV}$ energy band. The resulting $3 \sigma$ upper limit for the average source flux during each campaign is $\sim 17$ mcrab $\left(1.5 \times 10^{-10}\right.$ ergs $\mathrm{cm}^{-2} \mathrm{~s}^{-1}$ ) varying between 9 and 24 mcrab, with an integrated upper limit of $\sim 5 \mathrm{mcrab}\left(4.3 \times 10^{-11} \mathrm{ergs} \mathrm{cm}^{-2}\right.$ $\mathrm{s}^{-1}$ ).

As said previously, for the two observing sessions in which GRO J1744-28 was detected, this source presented a very steep spectrum, reasonably well fitted with an optically thin thermal Bremsstrahlung model, as given by the expression

$$
\frac{d N}{d E} \propto E^{-1.4} \exp \left(\frac{-E}{k T}\right) .
$$

In order to have a better coverage of the spectral behavior of the source, we also analyzed the $R X T E$ archival data obtained on 1996 March 22 and 1997 March 18. The source was very bright during $R X T E /$ PCA observations; therefore, any possible contamination by the other sources in the PCA's field of view is negligible. The PCA data reduction was performed with the help of standard tools from LHEASOFT/FTOOLS 5.0.4 package. Both $R X T E /$ PCA and GRANAT/SIGMA spectra were approximated by a model which is typical for pulsars: power law with high-
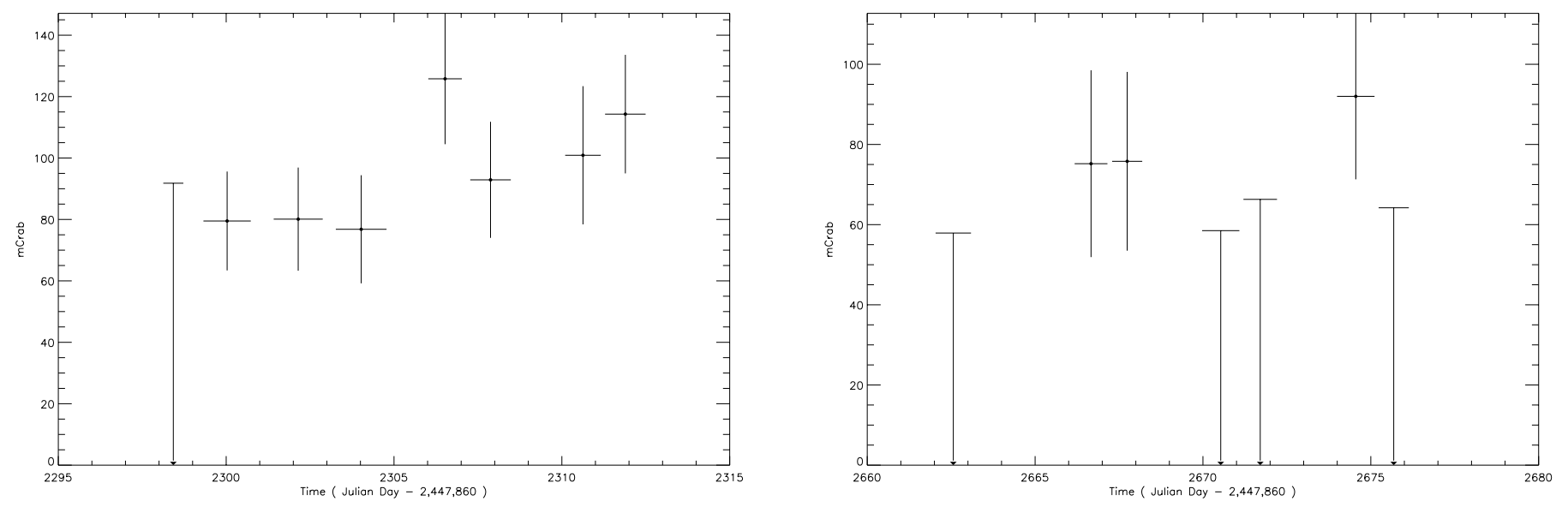

FIG. 2.- Light curve of GRO J1744-28 in the 35-75 keV energy band for the 1996 March and 1997 March campaigns, as observed with SIGMA. The horizontal axis corresponds to the date since the beginning of the SIGMA observations on 1989 December 1. 


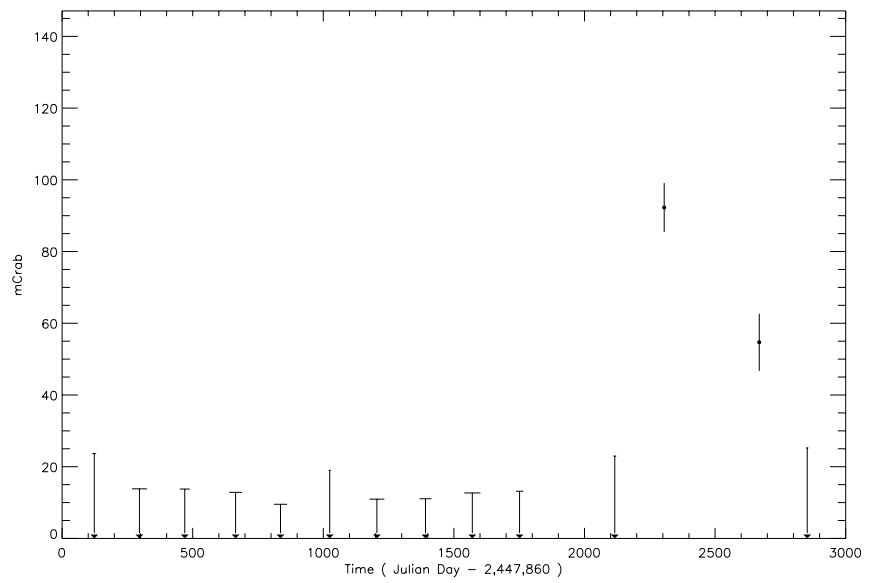

FIG. 3.- Light curve of GRO J1744-28 in the 35-75 keV energy band for all the SIGMA observing campaigns in the direction of the GC region, beginning on 1989 December 1.

energy cutoff, Gaussian line, and photoabsorption. The pure statistical significance of SIGMA data is much less than that of $R X T E /$ PCA. Because of this, the SIGMA spectral points almost do not influence on the parameters of the combined PCA + SIGMA spectral approximation. In order to make the spectral fit more sensitive to the SIGMA data, we artificially increased the uncertainties of the PCA spectral points to the value $5 \%$. The best-fit parameters of the combined PCA + SIGMA spectral fits are (for 1996/ 1997): photon index of the power law $\alpha=1.44 \pm 0.12 /$ $1.41 \pm 0.07 ; E_{\text {Hcutoff }}=14.7 \pm 0.7 / 16.4 \pm 0.9$. In Figure 4 we plot the 1-100 keV PCA/SIGMA spectrum corresponding to the 1996 March and 1997 March SIGMA observing periods, along with the SIGMA spectral fit.

Our result for the second outburst is compatible with the analysis of the same event of the source made on the
BATSE data and presented by Woods et al. (1999). For the epoch of the SIGMA observation, they report a temperature $k T \sim 14.5 \pm 1.8 \mathrm{keV}$ for the persistent emission (see Fig. 4, therein). However, as it will be shown later, there exists the possibility of a contamination of the BATSE data because of the presence of a transient source in the neighborhood of GRO J1744-28, namely GRS $1737-31$.

\section{CONCLUSION}

In the period going from 1990 to 1997 , observations of the GC region containing the coordinates of GRO J1744-28 were made with the SIGMA telescope in 14 opportunities, in March-April and September-October each year. In two of these opportunities, corresponding to 1996 March and 1997 March, GRO J1744-28 was clearly detected well above the $5 \sigma$ flux limit, corresponding to the two outbursts detected by several other instruments.

The mean fluxes for these two observations, as seen by SIGMA in the $35-75 \mathrm{keV}$ energy band, were 85 and 52 mcrab. In the $75-150 \mathrm{keV}$ energy band, the source was not detected. The spectra were reasonably well fitted by optically thin thermal Bremsstrahlung with $k T_{\text {Bremss }}=28$ and $18 \mathrm{keV}$, respectively. These values are compatible with that presented by Woods et al. (1999) for the second burst. However, the flux seen by SIGMA during this second observation campaign is almost a factor 7 smaller than that based on the BATSE observations of the region using the Earth-occultation technique. In this epoch, the transient source GRS 1737-31 was discovered with SIGMA (Sunyaev et al. 1997) and observed by RXTE and BeppoSAX/WFC (Marshall \& Smith 1997; Cui et al. 1997; Heise 1997). This source was active for a period of at least 1 month beginning on mid-February of 1997, in the 35-150 $\mathrm{keV}$ energy band, as reported by Trudolyubov et al. (1999). We argue that the presence of this source may explain, at least partially, the different flux values obtained with
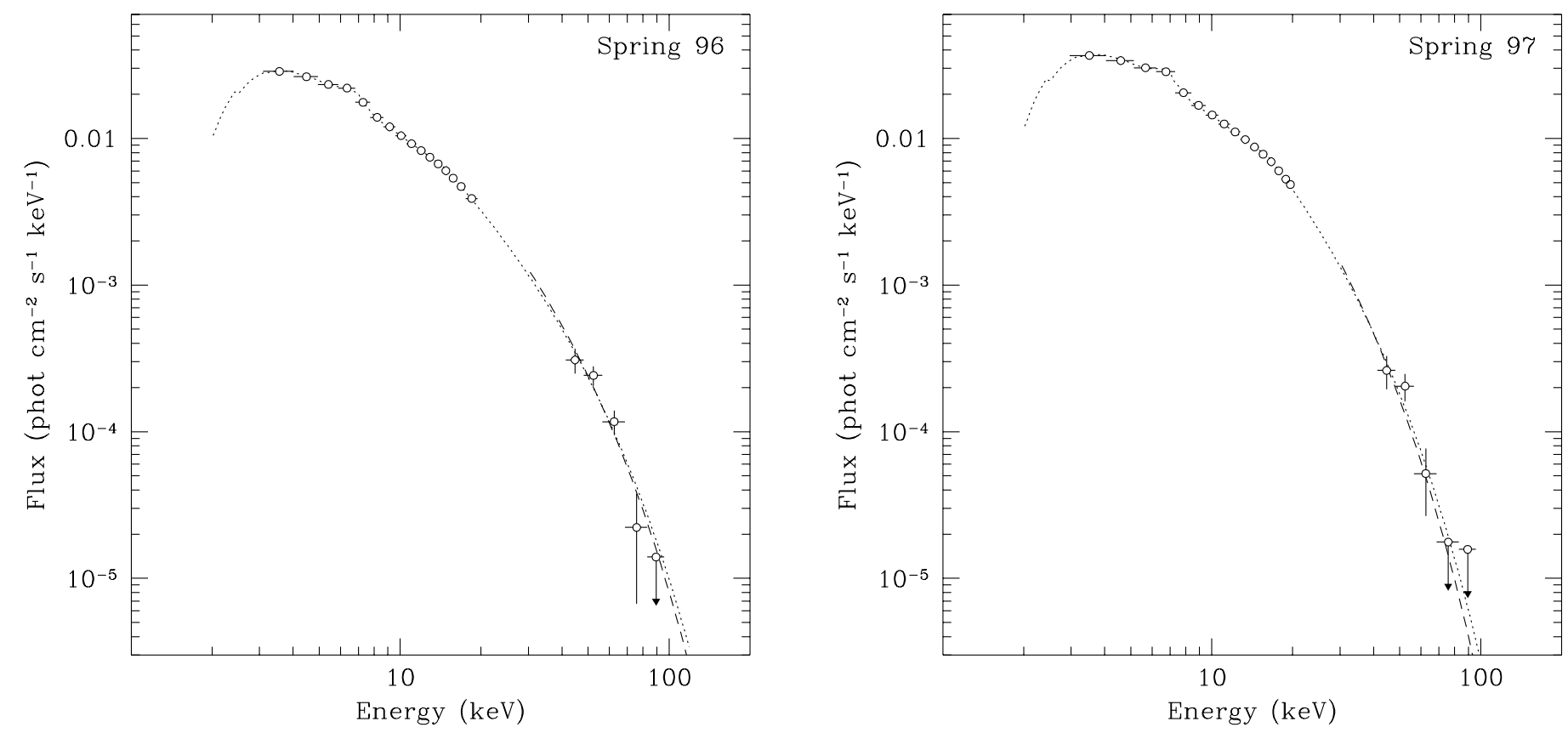

FIG. 4.-Combined RXTE/PCA and GRANAT/SIGMA spectra of GRO J1744-28 obtained in 1996 March and 1997 March. The PCA data points were renormalized to the SIGMA flux. The dotted line represents the best-fit model for the spectra (see text), with power-law photon index $\alpha \sim 1.4$ and high-energy cutoff $E \sim 15 \mathrm{keV}$ in the first case and $E \sim 16 \mathrm{keV}$ in the second. 
BATSE and SIGMA for the bursting pulsar. For GRS 1737-31, Trudolyubov et al. (1999) have found a flux of $\sim 100 \mathrm{mcrab}\left(1.6 \times 10^{-9} \mathrm{ergs} \mathrm{cm}^{-2} \mathrm{~s}^{-1}\right)$ in the $35-150 \mathrm{keV}$ band, which, added to the GRO J1744-28 flux $\left(4.5 \times 10^{-10}\right.$ ergs $\left.\mathrm{cm}^{-2} \mathrm{~s}^{-1}\right)$, accounts for a representative part of the flux reported from BATSE. It is important to note that the use of coded mask imaging techniques, like that used by SIGMA and other instruments of this kind, allows us to obtain in an unambiguous way the integrated flux and spectrum of the sources even in very crowded fields as in the case of the $\mathrm{GC}$ region.

With regard to the nature of the bursts, several authors have proposed that they are type II bursts (see, for instance, Lewin, Rutledge, \& Kommers 1996 for the first outburst). In their Figure 6, Woods et al. (1999) have shown that the parameter alpha, the ratio between the persistent flux and the average burst flux taken over the time interval since the previous burst, displayed for almost all the BATSE observations of the second outburst a value greater than 20 , which suggests that they are due to thermonuclear burning rather than spasmodic accretion (type I bursts rather than type II). However, if we consider the value of the flux obtained from the SIGMA observations as the sum of the persistent and the burst flux, and the value of the burst flux from the BATSE data, the value of alpha for the period of the observation is of the order of the 10 , more consistent with the type II nature of the bursts, as indicated by Lewin, van Paradijs, \& Taam (1995). Once again, the difference could be due to a possible contamination of the BATSE data by GRS $1737-31$.

We acknowledge the paramount contribution of the SIGMA Project Group of the CNES Toulouse Space Center to the overall success of the mission. We thank the staffs of the Lavotchine Space Company, of the Babakin Space Center, of the Baikonour Space Center, and the Evpatoria Ground Station for their unfailing support. This research has made use of data obtained through the High Energy Astrophysics Research Center Online Service, provided by the NASA/Goddard Space Flight Center. J. Mejía was supported by CAPES and CAPES grant BEX1224/000 . T. Villela was partially supported by CNPq under grants 302266/88-7-FA and 466184/00-0. The Space Research Institute (IKI) co-authors would like to acknowledge partial support of this work by RBRF grant 00-15-96649.
Augusteijn, T., et al. 1996, IAU Circ. 6369

Bildsten, L., \& Brown, E. F. 1997, ApJ, 477, 897

Bouchet, L., et al. 2001, ApJ, 548, 990

Cole, D. M., et al. 1997, ApJ, 480, 377

Cui, W., et al. 1997, IAU Circ. 6604

Daumerie, P. R., et al. 1996, Nature, 382, 141

Dotani, T., et al. 1996, IAU Circ. 6337

Finger, G. J., Wilson, R. B., \& van Paradijs, J. 1996, IAU Circ. 6286

Fishman, G. J., et al. 1995, IAU Circ. 6272

Heise, J. 1997, IAU Circ. 6606

Hurley, K., et al. 2000, ApJ, 537, 953

Kouveliotou, C., et al. 1996, IAU Circ. 6369

Lewin, W., Rutledge, R., \& Kommers, J. 1996, ApJ, 462, L39

\section{REFERENCES}

Lewin, W., van Paradijs, J., \& Taam, R. 1995, in X-Ray Binaries, ed. W. Lewin, J. van Paradijs, \& E. van de Heuvel (Cambridge: Cambridge Univ. Press), 196

Marshall, F. E., \& Smith, D. M. 1997, IAU Circ. 6603

Miller, G. S. 1996, ApJ, 468, L29

Nishiuchi, M., et al. 1999, ApJ, 517, 436

Paul, J., et al. 1991, Adv. Space Res., 11, 289

Sazonov, S. Y., Sunyaev, R. A., \& Lund, N. 1997, Astron. Lett., 23, 286

Sturner, S. J., \& Dermer, C. D. 1996, ApJ, 465, L31

Sunyaev, R., et al. 1997, IAU Circ. 6599

Trudolyubov, S., et al. 1999, A\&A, 342, 496

Woods, P. M., et al. 1999, ApJ, 517, 431 